\title{
State Estimation for Discrete-Time Takagi-Sugeno Fuzzy Systems with Time-Varying Delays
}

\author{
Ting Lei, ${ }^{1}$ Qiankun Song, ${ }^{2}$ and Yurong Liu ${ }^{3,4}$ \\ ${ }^{1}$ Department of Mathematics, Chongqing Jiaotong University, Chongqing 400074, China \\ ${ }^{2}$ School of Management, Chongqing Jiaotong University, Chongqing 400074, China \\ ${ }^{3}$ Department of Mathematics, Yangzhou University, Yangzhou 225002, China \\ ${ }^{4}$ Communication Systems and Networks (CSN) Research Group, Faculty of Engineering, King Abdulaziz University, \\ Jeddah 21589, Saudi Arabia
}

Correspondence should be addressed to Qiankun Song; qiankunsong@163.com

Received 25 August 2014; Accepted 10 September 2014

Academic Editor: Zidong Wang

Copyright (C) 2015 Ting Lei et al. This is an open access article distributed under the Creative Commons Attribution License, which permits unrestricted use, distribution, and reproduction in any medium, provided the original work is properly cited.

The state estimation problem is investigated for discrete-time Takagi-Sugeno fuzzy systems with time-varying delays. By constructing appropriate Lyapunov-Krasovskii functionals and employing matrix inequality technique, a delay-dependent linear matrix inequalities (LMIs) criterion is developed to estimate the systems state with some observed output measurements such that the error-state system is globally asymptotically stable. An example with simulations is given to show the effectiveness of the proposed criterion.

\section{Introduction}

It is known that many physical systems and processes have severe nonlinearities, which bring difficulties to the analysis and synthesis of the underlying systems $[1,2]$. As a powerful means to deal with complex nonlinear systems, fuzzy models have attracted rapidly growing attentions over the past decades [3]. Among various model-based fuzzy control approaches, Takagi-Sugeno (T-S) model has been an active topic due to the fact that it can combine the flexibility of fuzzy logic theory and fruitful linear system theory into a unified framework to approximate complex nonlinear systems [4]. In practice, time-delays often occur in many dynamic systems such as chemical processes, metallurgical processes, biological systems, and neural networks [5]. The existence of time-delays is usually a source of instability and poor performance [6]. Recently, many important results have been published on various analysis aspects for T-S models with time-delays. In particular, the stability, synchronization, and passivity have been intensively investigated; for example, see [7-27] and references therein.
On the other hand, state estimation is the process of assigning a value to an unknown system state variable based on measurements collected from the system [28]. Because of the complexity of large-scale systems, it is often the case that only partial information about the states of the nodes is available in the system outputs [29]. In order to understand the T-S fuzzy systems better, it becomes necessary to estimate the states of the nodes through available measurements [30].

Recently, some authors have studied the state estimation problem for T-S fuzzy systems with or without delays; for example, see [31-36] and references therein. In [31], a novel fuzzy-observer-design technique was developed for T-S fuzzy systems with unknown output disturbances. The system state and output disturbance were estimated asymptotically and simultaneously. In [32], the authors investigated the problem of robust $H_{\infty}$ state estimation for a class of multichannel networked nonlinear systems with multiple packet dropouts. The nonlinear plant was represented by T-S fuzzy-affine dynamic models with norm-bounded uncertainties, and stochastic variables with general probability distributions were adopted to characterize the data missing phenomenon in output 
channels. An admissible state estimator guaranteeing the stochastic stability of the resulting estimation error system with a prescribed $H_{\infty}$ disturbance attenuation level was designed. In [33], the authors proposed a novel fuzzy-based particle filter to reduce continuous state estimation errors due to failures in mode detection. It was fulfilled by considering a fuzzified contribution of each feasible mode in overall estimation. Two new resampling strategies were presented to tackle the degeneracy problem. A set of simulation test studies are conducted to extract the characteristic features and evaluate the performance of the proposed algorithm compared to observation and transition-based most likely modes tracking particle filter as one of the most meticulous proposed estimation algorithms. In [34], the T-S fuzzy model representation is extended to the state estimation of uncertain Markovian jumping Hopfield neural networks with mixed interval timevarying delays. Based on the Lyapunov-Krasovskii functional and stochastic analysis approach, several delay-dependent robust state estimators for such T-S fuzzy Markovian jumping Hopfield neural networks were achieved by solving a LMI. In [35], a design scheme for the state estimator for T-S fuzzy delayed Hopfield neural networks that uses strict output passivation of the error system was presented. Based on Lyapunov-Krasovskii functional, Jensens inequality, and linear matrix inequality (LMI) formulation, a delay-dependent criterion was proposed such that it makes the resulting estimation error system exponentially stable and passive from the input vector to the output error vector. In [36], the authors were concerned with the mixed $H_{\infty}$ and passivity based state estimation for a class of discrete-time fuzzy neural networks with the estimator gain change. Based on the Markovian system approach and linear matrix inequality technique, a new sufficient condition was derived such that the estimation error system was exponentially stable in the mean square sense. The estimator parameter was then determined by solving a set of LMIs.

It should be pointed out that most of aforementioned literatures on state estimation of T-S fuzzy systems have been concerned with the continuous-time systems only. To date, there have been very few results on the state estimation problems of discrete-time T-S fuzzy systems with delays [37]. In this paper, we continue to study the state estimation problems for discrete-time T-S fuzzy systems with time-varying delay. By employing appropriate Lyapunov-Krasovskii functionals and matrix inequality technique, we obtain a new delaydependent LMI sufficient condition such that estimation errors are globally asymptotically stable for discrete-time T-S fuzzy systems with time-varying delay.

Notations. The notations are quite standard. Throughout this paper, $I$ represents the unitary matrix with appropriate dimensions; $\mathbb{N}$ stands for the set of nonnegative integers; $\mathbb{R}^{n}$ and $\mathbb{R}^{n \times m}$ denote, respectively, the $n$-dimensional Euclidean space and the set of all $n \times m$ real matrices. The superscript " $T$ " denotes matrix transposition and the asterisk “*” denotes the elements below the main diagonal of a symmetric block matrix. The notation $X \geq Y$ (resp., $X>Y$ ) means that $X$ and $Y$ are symmetric matrices and that $X-Y$ is positive semidefinite (resp., positive definite). $\|\cdot\|$ is the Euclidean norm in
$\mathbb{R}^{n} . \lambda_{\min }(A)$ (resp., $\left.\lambda_{\max }(A)\right)$ denotes the least (resp., largest) eigenvalue of symmetric matrix $A$. For a positive constant $a,[a]$ denotes the integer part of $a$. For integers $a, b$ with $a<b, \mathbb{N}[a, b]$ denotes the discrete interval given by $\mathbb{N}[a, b]=$ $\{a, a+1, \ldots, b-1, b\} . C\left(\mathbb{N}[-\tau, 0], \mathbb{R}^{n}\right)$ denotes the set of all functions $\phi: \mathbb{N}[-\tau, 0] \rightarrow \mathbb{R}^{n} . \Delta V(k)$ denotes the difference of function $V(k)$ given by $\Delta V(k)=V(k+1)-V(k)$. Matrices, if not explicitly specified, are assumed to have compatible dimensions.

\section{Model Description and Preliminaries}

In this section, we consider a discrete-time T-S fuzzy system with time-varying delay with the $i$ th rule formulated in the following form.

Plant Rule i. IF $z_{1}(k)$ is $M_{i 1}$ and $\ldots$ and $z_{p}(k)$ is $M_{i p}$, THEN

$$
\begin{aligned}
x(k+1) & =A_{i} x(k)+B_{i} x(k-\tau(k))+U_{i} J(k), \\
y(k) & =C_{i} x(k)+D_{i} x(k-\tau(k)), \\
x(k) & =\varphi(k), \quad k \in \mathbb{N}[-\bar{\tau}, 0],
\end{aligned}
$$

for $k \in \mathbb{N}, i=1,2, \ldots, r$, and $r$ is the number of IF-THEN rules, where $x(k) \in \mathbb{R}^{n}$ is the state vector; $J(k) \in \mathbb{R}^{m}$ is a deterministic exogenous input; $y(k) \in \mathbb{R}^{m}$ is the measurement output vector; $A_{i}, B_{i}, U_{i}, C_{i}$, and $D_{i}$ are system matrices with compatible dimensions; the positive integer $\tau(k)$ corresponds to the transmission delay and satisfies $\tau \leq \tau(k) \leq$ $\bar{\tau}$, where $\tau \geq 0$ and $\bar{\tau} \geq 0$ are known integers.

Let $\mu_{i}(k)$ be the normalized membership function of the inferred fuzzy set $\gamma_{i}(k)$; that is,

$$
\mu_{i}(k)=\frac{\gamma_{i}(k)}{\sum_{i=1}^{r} \gamma_{i}(k)},
$$

where $\gamma_{i}(k)=\prod_{j=1}^{p} M_{i j}\left(z_{j}(k)\right)$ with $M_{i j}\left(z_{j}(k)\right)$ being the grade of membership function of $z_{j}(k)$ in $M_{i j}(k)$. It is assumed that $\gamma_{i}(k) \geq 0(i=1,2, \ldots, r)$ and $\sum_{i=1}^{r} \gamma_{i}(k)>0$ for all $k$. Thus, $\mu_{i}(k) \geq 0$ and $\sum_{i=1}^{r} \mu_{i}(k)=1$ for all $k$. And the T-S fuzzy model (1) can be represented as

$$
\begin{aligned}
x(k+1) & =\sum_{i=1}^{r} \mu_{i}(k)\left[A_{i} x(k)+B_{i} x(k-\tau(k))+U_{i} J(k)\right] \\
y(k) & =\sum_{i=1}^{r} \mu_{i}(k)\left[C_{i} x(k)+D_{i} x(k-\tau(k))\right] \\
x(k) & =\varphi(k), \quad k \in \mathbb{N}[-\bar{\tau}, 0]
\end{aligned}
$$

For the discrete-time T-S fuzzy systems with time-varying delay (1), we construct the full-order state estimation as follows. 
Estimator Rule i. IF $z_{1}(k)$ is $M_{i 1}$ and $\ldots$ and $z_{p}(k)$ is $M_{i p}$, THEN

$$
\begin{aligned}
\widehat{x}(k+1)= & A_{i} \widehat{x}(k)+B_{i} \hat{x}(k-\tau(k))+U_{i} J(k) \\
& +K(y(k)-\widehat{y}(k)), \\
\widehat{y}(k)= & C_{i} \widehat{x}(k)+D_{i} \widehat{x}(k-\tau(k)), \\
\widehat{x}(k)= & \psi(k), \quad k \in \mathbb{N}[-\bar{\tau}, 0],
\end{aligned}
$$

for $k \in \mathbb{N}$, where $\widehat{x}(k) \in \mathbb{R}^{n}$ is an estimation of the state $x(t)$ in model (1), $\widehat{y}(k) \in \mathbb{R}^{n}$ is the output vector of the state estimator, and $K \in \mathbb{R}^{n \times m}$ is the gain matrix of the state estimator to be designed. Using a standard fuzzy inference method, the fuzzy state estimator (4) is inferred as follows:

$$
\begin{aligned}
& \widehat{x}(k+1)= \sum_{i=1}^{r} \mu_{i}(k)\left[A_{i} \widehat{x}(k)+B_{i} \widehat{x}(k-\tau(k))\right. \\
&\left.+U_{i} J(k)+K(y(k)-\widehat{y}(k))\right], \\
& \widehat{y}(k)= \sum_{i=1}^{r} \mu_{i}(k)\left[C_{i} \widehat{x}(k)+D_{i} \hat{x}(k-\tau(k))\right], \\
& \widehat{x}(k)=\psi(k), \quad k \in \mathbb{N}[-\bar{\tau}, 0] .
\end{aligned}
$$

Let the error state be $e(k)=x(k)-\widehat{x}(k)$; then it follows from (3) and (5) that

$$
\begin{gathered}
e(k+1)=\sum_{i=1}^{r} \mu_{i}(k)\left[\left(-K C_{i}+A_{i}\right) e(k)\right. \\
\left.+\left(-K D_{i}+B_{i}\right) e(k-\tau(k))\right], \\
e(k)=\varphi(k)-\psi(k), \quad k \in \mathbb{N}[-\bar{\tau}, 0] .
\end{gathered}
$$

$$
\Pi^{(i)}=\left[\begin{array}{cccccc}
\Pi_{11} & \Pi_{12}^{(i)} & \Pi_{13}^{(i)} & 0 & 0 & 0 \\
* & \Pi_{22}^{(i)} & \Pi_{23}^{(i)} & P_{5} & P_{6} & \frac{2}{\bar{\tau}+\tau+1} P_{7} \\
* & * & -P_{4} & 0 & 0 & 0 \\
* & * & * & -P_{2}-P_{5} & 0 & 0 \\
* & * & * & * & -P_{3}-P_{6} & 0 \\
* & * & * & * & * & -\frac{2}{(\bar{\tau}-\tau)(\bar{\tau}+\tau+1)} P_{7}
\end{array}\right]<0
$$

Definition 1. The system (5) is said to be a globally asymptotically state estimated system of the system (3) if the estimation error system (6) is globally asymptotically stable.

In obtaining the main result of this paper, the following lemma will be useful for the proof, which can be proved by using the methods in [38].

Lemma 2. For any positive definite matrix $P \in \mathbb{R}^{n \times n}$, four integers $a, b, c$, and $d$ satisfying $c<d$ and $a+d<b$, and a vector function $x(s):[a+c, b] \rightarrow \mathbb{R}^{n}$, the following inequalities hold:

$$
\begin{aligned}
& -(d-c+1) \sum_{s=c}^{d} x^{T}(s) P x(s) \\
& \leq-\left(\sum_{s=c}^{d} x(s)\right)^{T} \times P\left(\sum_{s=c}^{d} x(s)\right) \\
& -(d-c+1)\left(b-a+1-\frac{c+d}{2}\right) \sum_{s=c}^{d} \sum_{u=s+a}^{b} x^{T}(u) P x(u) \\
& \leq-\left(\sum_{s=c}^{d} \sum_{u=s+a}^{b} x(u)\right)^{T} P\left(\sum_{s=c}^{d} \sum_{u=s+a}^{b} x(u)\right) .
\end{aligned}
$$

Remark 3. Let $c=k-h_{M}$ and $d=k-h_{m}-1$; then the first inequality of Lemma 2 in this paper turns into the first inequality of Lemma 1 in [38]. If we let $c=-h_{M}, d=-h_{m}, a=$ $k$, and $b=k-h_{m}-1$, then the second inequality of Lemma 2 in this paper turns into the second inequality of Lemma 1 in [38]. So, Lemma 2 in this paper is more general than Lemma 1 in [38].

\section{Main Results}

In this section, we will establish our main criterion based on the LMI approach.

Theorem 4. If there exist seven symmetric positive definite matrices $P_{i}(i=1,2,3,4,5,6,7)$ and a matrix $Q$ such that the following LMIs hold for $i=1,2, \ldots, r$,

(5) is a globally asymptotically state estimated system of system (3), and the estimator gain matrix $K$ can be designed as

$$
K=P_{1}^{-1} Q
$$

in which $\Pi_{11}=-P_{1}+\tau^{2} P_{5}+\bar{\tau}^{2} P_{6}+((\bar{\tau}-\tau)(\bar{\tau}+\tau+1) / 2) P_{7}, \Pi_{12}^{(i)}=$ $-P_{1}-Q C_{i}+P_{1} A_{i}, \Pi_{13}^{(i)}=-Q D_{i}+P_{1} B_{i}, \Pi_{22}^{(i)}=-2 P_{1}-Q C_{i}-$ $C_{i}^{T} Q^{T}+P_{1} A_{i}+A_{i}^{T} P_{1}+P_{2}+P_{3}+(1+\bar{\tau}-\tau) P_{4}-P_{5}-P_{6}-$ $(2(\bar{\tau}-\tau) /(\bar{\tau}+\tau+1)) P_{7}$, and $\Pi_{23}^{(i)}=-Q D_{i}+P_{1} B_{i}$, then system 
Proof. Defining $\eta(k)=e(k+1)-e(k)$, we consider the following Lyapunov-Krasovskii functional candidate for model (6) as

$$
V(k)=\sum_{i=1}^{5} V_{i}(k)
$$

where

$$
\begin{aligned}
& V_{1}(k)=e^{T}(k) P_{1} e(k), \\
& V_{2}(k)=\sum_{i=k-\tau}^{k-1} e^{T}(i) P_{2} e(i)+\sum_{i=k-\bar{\tau}}^{k-1} e^{T}(i) P_{3} e(i), \\
& V_{3}(k)=\sum_{i=k-\tau(k)}^{k-1} e^{T}(i) P_{4} e(i)+\sum_{l=k-\bar{\tau}+1}^{k-\tau} \sum_{i=l}^{k-1} e^{T}(i) P_{4} e(i), \\
& V_{4}(k)=\tau \sum_{l=k-\tau}^{k-1} \sum_{i=l}^{k-1} \eta^{T}(i) P_{5} \eta(i)+\bar{\tau} \sum_{l=k-\bar{\tau}}^{k-1} \sum_{i=l}^{k-1} \eta^{T}(i) P_{6} \eta(i), \\
& V_{5}(k)=\sum_{l=\tau+1}^{\bar{\tau}} \sum_{j=1}^{l} \sum_{i=k-j}^{k-1} \eta^{T}(i) P_{7} \eta(i) .
\end{aligned}
$$

Calculating the difference of $V_{i}(k)(i=1,2,3,4,5)$ along the trajectories of model (6), we obtain that

$$
\begin{aligned}
\Delta V_{1}(k)= & e^{T}(k+1) P_{1} e(k+1)-e^{T}(k) P_{1} e(k) \\
= & -\eta^{T}(k) P_{1} \eta(k)+2 \eta^{T}(k) P_{1}(e(k+1)-e(k)) \\
& +2 e^{T}(k) P_{1}(e(k+1)-e(k)) \\
= & -\eta^{T}(k) P_{1} \eta(k)+2 \eta^{T}(k) P_{1} \\
& \times \sum_{i=1}^{r} \mu_{i}(k)\left[\left(-I-K C_{i}+A_{i}\right) e(k)\right. \\
& \left.\quad+\left(-K D_{i}+B_{i}\right) e(k-\tau(k))\right] \\
& +2 e^{T}(k) P_{1} \\
& \times \sum_{i=1}^{r} \mu_{i}(k)\left[\left(-I-K C_{i}+A_{i}\right) e(k)\right. \\
& \left.+\left(-K D_{i}+B_{i}\right) e(k-\tau(k))\right] \\
= & \sum_{i=1}^{r} \mu_{i}(k)\left[-\eta^{T}(k) P_{1} \eta(k)\right. \\
& +2 \eta^{T}(k)\left(-P_{1}-P_{1} K C_{i}+P_{1} A_{i}\right) e(k) \\
& +2 \eta^{T}(k)\left(-P_{1} K D_{i}+P_{1} B_{i}\right) e(k-\tau(k)) \\
& +2 e^{T}(k)\left(-P_{1}-P_{1} K C_{i}+P_{1} A_{i}\right) e(k) \\
& \left.+2 e^{T}(k)\left(-P_{1} K D_{i}+P_{1} B_{i}\right) e(k-\tau(k))\right],
\end{aligned}
$$

$$
\begin{aligned}
\Delta V_{2}(k)= & e^{T}(k)\left(P_{2}+P_{3}\right) e(k)-e^{T}(k-\tau) P_{2} e(k-\tau) \\
& -e^{T}(k-\bar{\tau}) P_{3} e(k-\bar{\tau}), \\
\Delta V_{3}(k)= & \sum_{i=k+1-\tau(k+1)}^{k-\tau} e^{T}(i) P_{4} e(i)
\end{aligned}
$$

$$
\begin{aligned}
& +\sum_{i=k-\tau+1}^{k-1} e^{T}(i) P_{4} e(i)+e^{T}(k) P_{4} e(k) \\
& -\sum_{i=k-\tau(k)+1}^{k-1} e^{T}(i) P_{4} e(i)-e^{T}(k-\tau(k)) \\
& \times P_{4} e(k-\tau(k)) \\
& +(\bar{\tau}-\tau) e^{T}(k) P_{4} e(k)-\sum_{i=k-\bar{\tau}+1}^{k-\tau} e^{T}(i) P_{4} e(i) \\
\leq & (1+\bar{\tau}-\tau) e^{T}(k) P_{4} e(k) \\
& -e^{T}(k-\tau(k)) P_{4} e(k-\tau(k)) \\
\Delta V_{4}(k)= & \eta^{T}(k)\left(\tau^{2} P_{5}+\bar{\tau}^{2} P_{6}\right) \eta(k) \\
& -\tau \sum_{i=k-\tau}^{k-1} \eta^{T}(i) P_{5} \eta(i)-\bar{\tau} \sum_{i=k-\bar{\tau}}^{k-1} \eta^{T}(i) P_{6} \eta(i), \\
\Delta V_{5}(k)= & \sum_{l=\tau+1}^{\bar{\tau}} \sum_{j=1}^{l}\left[\eta^{T}(k) P_{7} \eta(k)-\eta^{T}(k-j) P_{7} \eta(k-j)\right] \\
= & \frac{(\bar{\tau}-\tau)(\bar{\tau}+\tau+1)}{2} \eta^{T}(k) P_{7} \eta(k) \\
& -\sum_{l=-\bar{\tau} i=l+k}^{-\tau-1} \sum^{k-1} \eta^{T}(i) P_{7} \eta(i) .
\end{aligned}
$$

In deriving inequality (14), the conditions $\tau \leq \tau(k)$ and $\tau(k+1) \leq \bar{\tau}$ have been used.

From the first inequality of Lemma 2 , we have that

$$
\begin{gathered}
-\tau \sum_{i=k-\tau}^{k-1} \eta^{T}(i) P_{5} \eta(i)-\bar{\tau} \sum_{i=k-\bar{\tau}}^{k-1} \eta^{T}(i) P_{6} \eta(i) \\
\leq-\sum_{i=k-\tau}^{k-1} \eta^{T}(i) P_{5} \sum_{i=k-\tau}^{k-1} \eta(i)-\sum_{i=k-\bar{\tau}}^{k-1} \eta^{T}(i) P_{6} \sum_{i=k-\bar{\tau}}^{k-1} \eta(i) \\
=\left[\begin{array}{c}
e(k) \\
e(k-\tau)
\end{array}\right]^{T}\left[\begin{array}{cc}
-P_{5} & P_{5} \\
* & -P_{5}
\end{array}\right]\left[\begin{array}{c}
e(k) \\
e(k-\tau)
\end{array}\right] \\
+\left[\begin{array}{c}
e(k) \\
e(k-\bar{\tau})
\end{array}\right]^{T}\left[\begin{array}{cc}
-P_{6} & P_{6} \\
* & -P_{6}
\end{array}\right]\left[\begin{array}{c}
e(k) \\
e(k-\bar{\tau})
\end{array}\right] .
\end{gathered}
$$


From the second inequality of Lemma 2, we can get that

$$
\begin{aligned}
- & \sum_{l=-\bar{\tau}}^{-\tau-1} \sum_{i=l+k}^{k-1} \eta^{T}(i) P_{7} \eta(i) \\
\leq & -\frac{2}{(\bar{\tau}-\tau)(\bar{\tau}+\tau+1)}\left[\sum_{l=-\bar{\tau}}^{-\tau-1} \sum_{i=l+k}^{k-1} \eta(i)\right]^{T} \\
& \times P_{7}\left(\sum_{l=-\bar{\tau}}^{-\tau-\tau} \sum_{i=l+k}^{k-1} \eta(i)\right) \\
= & -\frac{2}{(\bar{\tau}-\tau)(\bar{\tau}+\tau+1)}\left[(\bar{\tau}-\tau) e(k)-\sum_{i=k-\bar{\tau}}^{k-\tau-1} e(i)\right]^{T} \\
& \times P_{7}\left[(\bar{\tau}-\tau) e(k)-\sum_{i=k-\bar{\tau}}^{k-\tau-1} e(i)\right] \\
= & -\frac{2(\bar{\tau}-\tau)}{\bar{\tau}+\tau+1} e^{T}(k) P_{7} e(k) \\
& +\frac{4}{\bar{\tau}+\tau+1} e^{T}(k) P_{7} \sum_{i=k-\bar{\tau}}^{k-\tau-1} e(i) \\
& \left.-\frac{2}{(\bar{\tau}-\tau)(\bar{\tau}+\tau+1)}\left[\sum_{i=k-\bar{\tau}}^{k-\tau-1} e(i)\right]^{T} P_{i=k-\bar{\tau}}^{k-\tau-1} e(i)\right] .
\end{aligned}
$$

Denote $\alpha(k)=\left[\eta^{T}(k), e^{T}(k), e^{T}(k-\tau(k)), e^{T}(k-\tau), e^{T}(k-\right.$ $\left.\bar{\tau}), \sum_{i=k-\bar{\tau}}^{k-\tau-1} e^{T}(i)\right]^{T}$. It follows from (9) to (18) that

$$
\begin{aligned}
& \Delta V(k) \leq \sum_{i=1}^{r} \mu_{i}(k) \\
& \times\left\{\eta ^ { T } ( k ) \left(-P_{1}+\tau^{2} P_{5}+\bar{\tau}^{2} P_{6}\right.\right. \\
& \left.+\frac{(\bar{\tau}-\tau)(\bar{\tau}+\tau+1)}{2} P_{7}\right) \eta(k) \\
& +2 \eta^{T}(k)\left(-P_{1}-P_{1} K C_{i}+P_{1} A_{i}\right) e(k) \\
& +2 \eta^{T}(k)\left(-P_{1} K D_{i}+P_{1} B_{i}\right) e(k-\tau(k)) \\
& +e^{T}(k)\left(-2 P_{1}-2 P_{1} K C_{i}+2 P_{1} A_{i}+P_{2}\right. \\
& +P_{3}+(1+\bar{\tau}-\tau) P_{4}-P_{5}-P_{6} \\
& \left.-\frac{2(\bar{\tau}-\tau)}{\bar{\tau}+\tau+1} P_{7}\right) e(k) \\
& +2 e^{T}(k)\left(-P_{1} K D_{i}+P_{1} B_{i}\right) e(k-\tau(k)) \\
& +2 e^{T}(k) P_{5} e(k-\tau)+2 e^{T}(k) P_{6} e(k-\bar{\tau})
\end{aligned}
$$

$$
\begin{gathered}
+\frac{4}{\bar{\tau}+\tau+1} e^{T}(k) P_{7} \sum_{i=k-\bar{\tau}}^{k-\tau-1} e(i) \\
-e^{T}(k-\tau(k)) P_{4} e(k-\tau(k)) \\
+e^{T}(k-\tau)\left(-P_{2}-P_{5}\right) e^{T}(k-\tau) \\
+e^{T}(k-\bar{\tau})\left(-P_{3}-P_{6}\right) e^{T}(k-\bar{\tau}) \\
-\frac{2}{(\bar{\tau}-\tau)(\bar{\tau}+\tau+1)} \\
\left.\times\left[\sum_{i=k-\bar{\tau}}^{k} e(i)\right]^{T} P_{7}\left[\sum_{i=k-\bar{\tau}}^{k-\tau-1} e(i)\right]\right\} \\
=\sum_{i=1}^{r} \mu_{i}(k) \alpha^{T}(k) \Pi^{(i)} \alpha(k) .
\end{gathered}
$$

From (8) and (19), we know that the system (5) is a globally asymptotically state estimated system of system (3). The proof is completed.

Remark 5. In order to reduce the conservatism of the obtained result, the triple-summable term in $V_{5}(k)$ is added, which has been used in literature [38].

\section{Example}

To verify the effectiveness of the theoretical result of this paper, we consider the following example.

Example 1. Consider a T-S fuzzy system (1) with $r=2$, where $r$ is the number of IF-THEN rules. The time-varying delay $\tau(k)=2+\sin (k \pi / 2)$ with $\tau=1$ and $\bar{\tau}=3$, and the deterministic exogenous input $J(k)=(-3 \sin (k \pi / 2), 2 \cos (k \pi / 2))^{T}$. Other parameters are given as follows:

$$
\begin{aligned}
A_{1} & =\left[\begin{array}{cc}
0.9 & 0.1 \\
-0.02 & 0.7
\end{array}\right], & A_{2} & =\left[\begin{array}{cc}
0.1 & -0.2 \\
-0.01 & 0.8
\end{array}\right], \\
B_{1} & =\left[\begin{array}{cc}
0.01 & 0.02 \\
-0.03 & 0.15
\end{array}\right], & B_{2} & =\left[\begin{array}{cc}
-0.01 & 0.02 \\
0.01 & 0.09
\end{array}\right], \\
C_{1} & =\left[\begin{array}{cc}
-0.01 & 0.02 \\
0.01 & 0.03
\end{array}\right], & C_{2} & =\left[\begin{array}{cc}
0.01 & -0.01 \\
0.02 & 0.01
\end{array}\right], \\
D_{1} & =\left[\begin{array}{cc}
0.02 & 0.03 \\
-0.02 & 0.01
\end{array}\right], & D_{2} & =\left[\begin{array}{cc}
-0.01 & -0.03 \\
0.01 & -0.02
\end{array}\right], \\
U_{1} & =\left[\begin{array}{cc}
-0.08 & 0.07 \\
0.01 & 0.04
\end{array}\right], & U_{2} & =\left[\begin{array}{cc}
0.02 & -0.09 \\
0.01 & -0.04
\end{array}\right] .
\end{aligned}
$$

The fuzzy membership functions are taken as $\mu_{1}(k)=$ $\sin ^{2}\left(3 x_{1}(k)\right)$ and $\mu_{2}(k)=\cos ^{2}\left(3 x_{1}(k)\right)$. 


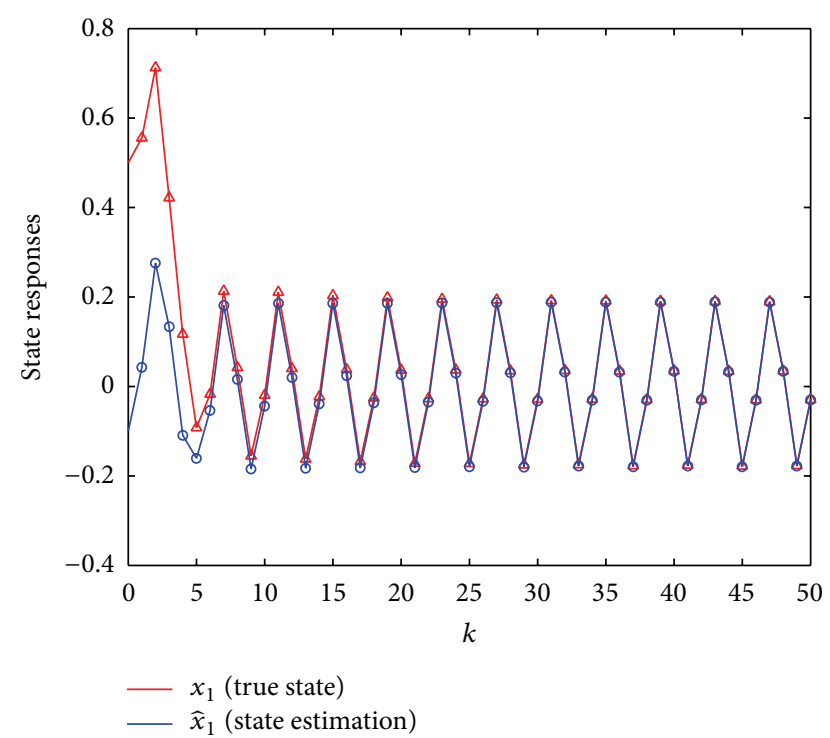

FIGURE 1: The responses of the true state $x_{1}(k)$ (red triangle) and its estimation $y_{1}(k)$ (blue circle).

By using the Matlab LMI Control Toolbox, we can find a solution to the LMIs in (8) as follows:

$$
\begin{array}{cc}
P_{1}=\left[\begin{array}{cc}
138.5930 & 12.7196 \\
12.7196 & 262.8188
\end{array}\right], & P_{2}=\left[\begin{array}{ll}
5.2911 & 0.4015 \\
0.4015 & 5.3830
\end{array}\right], \\
P_{3}=\left[\begin{array}{ll}
8.9596 & 0.9476 \\
0.9476 & 6.6167
\end{array}\right], & P_{4}=\left[\begin{array}{cc}
3.7939 & -0.0013 \\
-0.0013 & 19.8495
\end{array}\right], \\
P_{5}=\left[\begin{array}{cc}
13.8548 & -0.1830 \\
-0.1830 & 17.5328
\end{array}\right], & P_{6}=\left[\begin{array}{cc}
0.4305 & -0.3519 \\
-0.3519 & 5.9357
\end{array}\right], \\
P_{7}=\left[\begin{array}{cc}
7.2292 & 0.2589 \\
0.2589 & 15.4451
\end{array}\right], & Q=\left[\begin{array}{cc}
56.6136 & 80.2187 \\
-94.4366 & 272.2139
\end{array}\right] .
\end{array}
$$

Subsequently, we can obtain from $K=P_{1}^{-1} Q$ that

$$
K=\left[\begin{array}{cc}
0.4434 & 0.4859 \\
-0.3808 & 1.0122
\end{array}\right]
$$

According to Theorem 4, we know that the system (5) is a globally asymptotically state estimated system of system (3). The simulation results are shown in Figures 1 and 2, which demonstrate the effectiveness of the developed approach for the design of the state estimator for discrete-time TakagiSugeno fuzzy systems with time-varying delays.

\section{Conclusions}

In this paper, the state estimation problem has been investigated for discrete-time T-S fuzzy systems with time-varying delays. By employing Lyapunov functional method and the matrix inequality techniques, a delay-dependent LMIs criterion has been established to estimate the systems state with some observed output measurements such that the errorstate system is globally asymptotically stable. An example

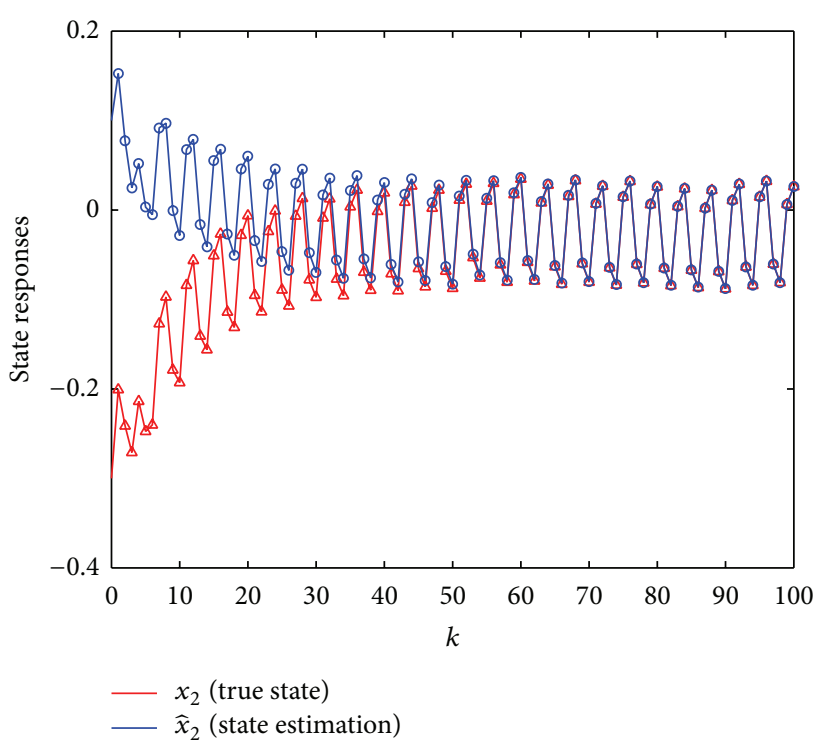

FIGURE 2: The responses of the true state $x_{2}(k)$ (red triangle) and its estimation $y_{2}(k)$ (blue circle).

has been provided to show the effectiveness of the proposed criterion.

\section{Conflict of Interests}

The authors declare that there is no conflict of interests regarding the publication of this paper.

\section{Acknowledgments}

The authors would like to thank the editor and the reviewers for their valuable suggestions and comments which have led to a much improved paper. This work was supported by the National Natural Science Foundation of China under Grants 61273021 and 11172247 and in part by the Natural Science Foundation Project of CQ cstc2013jjB40008.

\section{References}

[1] J. Hu, Z. Wang, B. Shen, and H. Gao, "Quantised recursive filtering for a class of nonlinear systems with multiplicative noises and missing measurements," International Journal of Control, vol. 86, no. 4, pp. 650-663, 2013.

[2] Z. Wang, H. Dong, B. Shen, and H. Gao, "Finite-horizon $H_{\infty}$ filtering with missing measurements and quantization effects," IEEE Transactions on Automatic Control, vol. 58, no. 7, pp. 17071718, 2013.

[3] G. Feng, "A survey on analysis and design of model-based fuzzy control systems," IEEE Transactions on Fuzzy Systems, vol. 14, no. 5, pp. 676-697, 2006.

[4] C. Lin, Q.-G. Wang, T. H. Lee, Y. He, and B. Chen, "Observerbased fuzzy control design for T-S fuzzy systems with state delays," Automatica, vol. 44, no. 3, pp. 868-874, 2008.

[5] H. Dong, Z. Wang, and H. Gao, "Distributed $H_{\infty}$ filtering for class of markovian jump nonlinear time-delay systems over 
lossy sensor networks," IEEE Transactions on Industrial Electronics, vol. 60, no. 10, pp. 4665-4672, 2013.

[6] Y. Li and X. Zhou, "T-S fuzzy model-based approximation and filter design for stochastic time-delay systems with Hankel norm criterion," Abstract and Applied Analysis, vol. 2014, Article ID 937495, 12 pages, 2014.

[7] H. K. Lam, "Stability analysis of T-S fuzzy control systems using parameter-dependent Lyapunov function," IET Control Theory \& Applications, vol. 3, no. 6, pp. 750-762, 2009.

[8] Z. Yang and Y.-P. Yang, "New delay-dependent stability analysis and synthesis of T-S fuzzy systems with time-varying delay," International Journal of Robust and Nonlinear Control, vol. 20, no. 3, pp. 313-322, 2010.

[9] C. Peng, L.-Y. Wen, and J.-Q. Yang, "On delay-dependent robust stability criteria for uncertain T-S fuzzy systems with interval time-varying delay," International Journal of Fuzzy Systems, vol. 13, no. 1, pp. 35-44, 2011.

[10] S. Lakshmanan, R. Rakkiyappan, and P. Balasubramaniam, "Global robust stability criteria for T-S fuzzy systems with distributed delays and time delay in the leakage term," Iranian Journal of Fuzzy Systems, vol. 9, no. 2, pp. 127-146, 2012.

[11] S. Xing, Q. Zhang, and Y. Zhang, "Finite-time stability analysis and control for a class of stochastic singular biological economic systems based on T-S fuzzy model," Abstract and Applied Analysis, vol. 2013, Article ID 946491, 10 pages, 2013.

[12] F. Ahmida and E. H. Tissir, "Exponential stability of uncertain T-S fuzzy switched systems with time delay," International Journal of Automation and Computing, vol. 10, no. 1, pp. 32-38, 2013.

[13] H.-B. Zeng, J. H. Park, J.-W. Xia, and S.-P. Xiao, "Improved delay-dependent stability criteria for T-S fuzzy systems with time-varying delay," Applied Mathematics and Computation, vol. 235, pp. 492-501, 2014.

[14] Y. Mao and H. Zhang, "Exponential stability and robust $H_{\infty}$ control of a class of discrete-time switched non-linear systems with time-varying delays via T-S fuzzy model," International Journal of Systems Science, vol. 45, no. 5, pp. 1112-1127, 2014.

[15] X. Yang, L. Wu, H.-K. Lam, and X. Su, "Stability and stabilization of discrete-time T-S fuzzy systems with stochastic perturbation and time-varying delay," IEEE Transactions on Fuzzy Systems, vol. 22, no. 1, pp. 124-138, 2014.

[16] C. Ahn and P. Kim, "T-S fuzzy adaptive delayed feedback synchronization for time-delayed chaotic systems with uncertain parameters," International Journal of Modern Physics B, vol. 25, no. 23-24, pp. 3253-3267, 2011.

[17] X. Wu, Y. Wang, X. Dang, and L. Huang, "Adaptive synchronization of T-S fuzzy complex networks with time-varying delays via the pinning control method," Nonlinear Dynamics, vol. 74, no. 1-2, pp. 143-152, 2013.

[18] D. Xu, W. Gui, P. Zhao, and C. Yang, "Hybrid synchronization of general T-S fuzzy complex dynamical networks with timevarying delay," Mathematical Problems in Engineering, vol. 2013, Article ID 384654, 8 pages, 2013.

[19] F.-H. Hsiao, "Optimal exponential synchronization of chaotic systems with multiple time delays via fuzzy control," Abstract and Applied Analysis, vol. 2013, Article ID 742821, 19 pages, 2013.

[20] J. Liang, Z. Wang, and X. Liu, "Robust passivity and passification of stochastic fuzzy time-delay systems," Information Sciences, vol. 180, no. 9, pp. 1725-1737, 2010.

[21] J. Liang, Z. Wang, and X. Liu, "On passivity and passification of stochastic fuzzy systems with delays: the discrete-time case,"
IEEE Transactions on Systems, Man, and Cybernetics Part B: Cybernetics, vol. 40, no. 3, pp. 964-969, 2010.

[22] B. Zhang, W.X. Zheng, and S. Xu, "Passivity analysis and passive control of fuzzy systems with time-varying delays," Fuzzy Sets and Systems, vol. 174, no. 1, pp. 83-98, 2011.

[23] C. Han, L. Wu, P. Shi, and Q. Zeng, "Passivity and passification of T-S fuzzy descriptor systems with stochastic perturbation and time delay," IET Control Theory \& Applications, vol. 7, no. 13, pp. 1711-1724, 2013.

[24] T. Lei, Z. Chen, Q. Song, and Z. Zhao, "Passivity analysis and passive control for T-S fuzzy systems with leakage delay and mixed time-varying delays," Abstract and Applied Analysis, vol. 2013, Article ID 524028, 9 pages, 2013.

[25] Q. Song, Z. Wang, and J. Liang, "Analysis on passivity and passification of T-S fuzzy systems with time-varying delays," Journal of Intelligent and Fuzzy Systems, vol. 24, no. 1, pp. 21-30, 2013.

[26] Q. Song, Z. Zhao, and J. Yang, "Passivity and passification for stochastic Takagi-Sugeno fuzzy systems with mixed timevarying delays," Neurocomputing, vol. 122, pp. 330-337, 2013.

[27] T. Lei, Q. Song, and Z. Zhao, "Further result on passivity for discrete-time stochastic T-S fuzzy systems with time-varying delays," Discrete Dynamics in Nature and Society, vol. 2014, Article ID 657621, 8 pages, 2014.

[28] D. Ding, Z. Wang, H. Dong, and H. Shu, "Distributed $H_{\infty}$ state estimation with stochastic parameters and nonlinearities through sensor networks: the finite-horizon case," Automatica, vol. 48, no. 8, pp. 1575-1585, 2012.

[29] B. Shen, Z. Wang, D. Ding, and H. Shu, " $H_{\infty}$ state estimation for complex networks with uncertain inner coupling and incomplete measurements," IEEE Transactions on Neural Networks and Learning Systems, vol. 24, no. 12, pp. 2027-2037, 2013.

[30] B.-K. Lee, K.-H. Lee, and B.-S. Chen, "State estimation of stochastic T-S fuzzy systems," International Journal of Fuzzy Systems, vol. 8, no. 1, pp. 46-56, 2006.

[31] Z. Gao, X. Shi, and S. X. Ding, "Fuzzy state/disturbance observer design for T-S fuzzy systems with application to sensor fault estimation," IEEE Transactions on Systems, Man, and Cybernetics Part B: Cybernetics, vol. 38, no. 3, pp. 875-880, 2008.

[32] J. Qiu, G. Feng, and H. Gao, "Nonsynchronized-State estimation of multichannel networked nonlinear systems with multiple packet dropouts Via TS Fuzzy-Affine dynamic models," IEEE Transactions on Fuzzy Systems, vol. 19, no. 1, pp. 75-90, 2011.

[33] K. Salahshoor and M. F. Samadi, "A novel fuzzy approach for state estimation of nonlinear hybrid systems," Asian Journal of Control, vol. 14, no. 4, pp. 974-990, 2012.

[34] X. Li and R. Rakkiyappan, "Robust asymptotic state estimation of Takagi-Sugeno fuzzy Markovian jumping Hopfield neural networks with mixed interval time-varying delays," Mathematical Methods in the Applied Sciences, vol. 34, no. 17, pp. 2197-2207, 2011.

[35] C. K. Ahn, "State estimation for T-S fuzzy Hopfield neural networks via strict output passivation of the error system," International Journal of General Systems, vol. 42, no. 5, pp. 503-518, 2013.

[36] D. Zhang, W. Cai, and Q.-G. Wang, "Mixed $H_{\infty}$ and passivity based state estimation for fuzzy neural networks with Markovian-type estimator gain change," Neurocomputing, vol. 139, pp. 321-327, 2014. 
[37] J. Carlos González-Castolo and E. López-Mellado, "Fuzzy state estimation of discrete event systems," in MICAI 2006: Advances in Artificial Intelligence, vol. 4293 of Lecture Notes in Computer Science, pp. 90-100, 2006.

[38] O. M. Kwon, M. J. Park, J. H. Park, S. M. Lee, and E. J. Cha, "New criteria on delay-dependent stability for discrete-time neural networks with time-varying delays," Neurocomputing, vol. 121, pp. 185-194, 2013. 


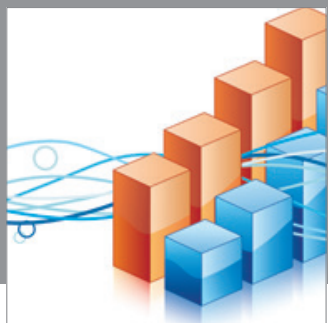

Advances in

Operations Research

mansans

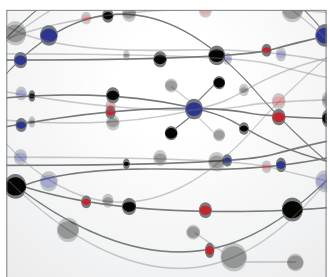

The Scientific World Journal
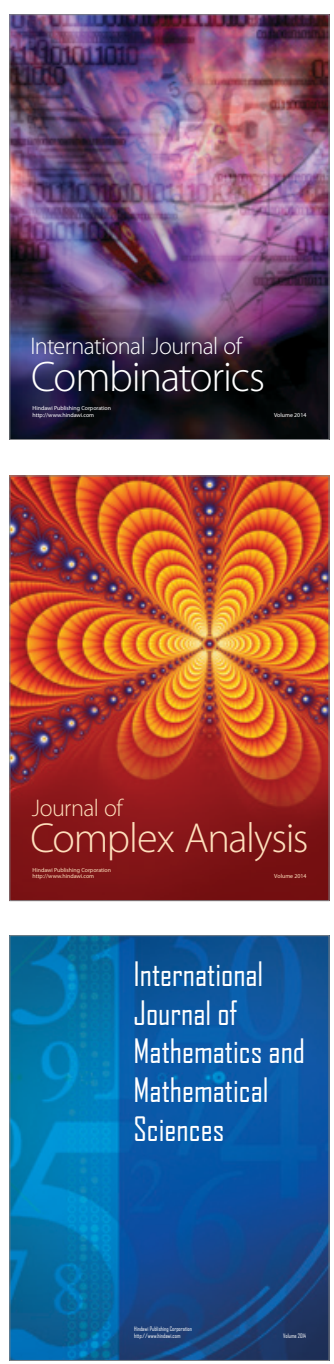
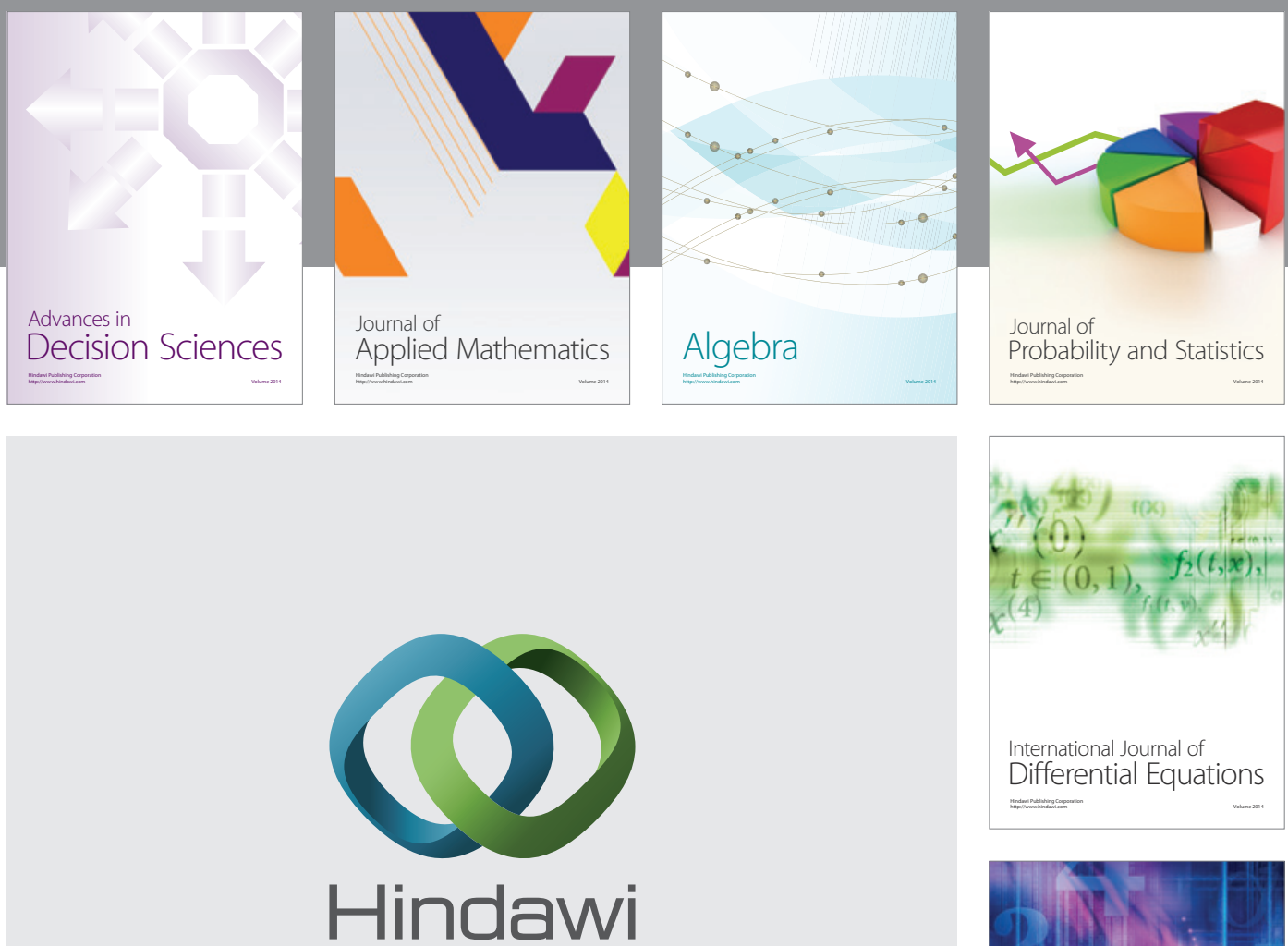

Submit your manuscripts at http://www.hindawi.com
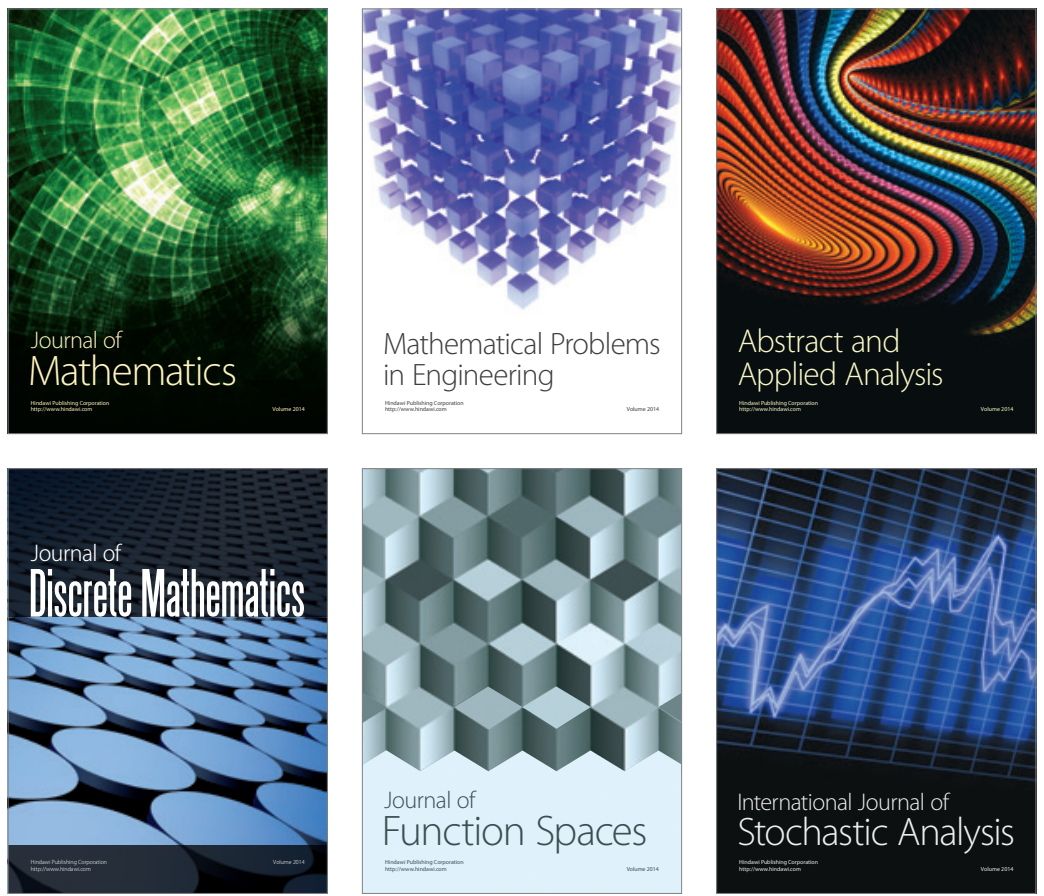

Journal of

Function Spaces

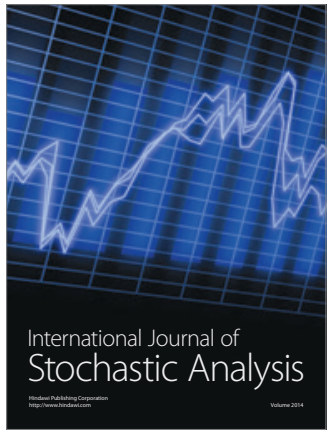

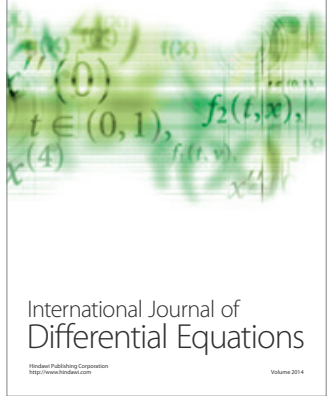
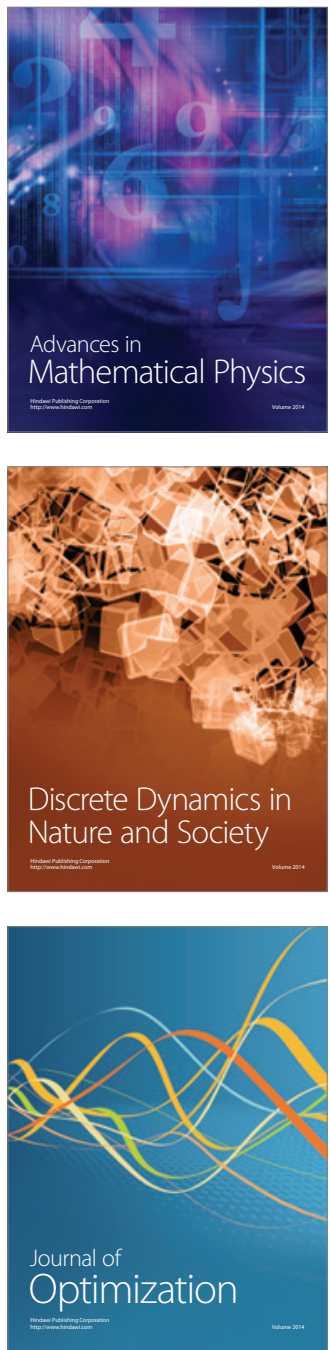